Revista Iberoamericana de las Ciencias Biológicas

y Agropecuarias

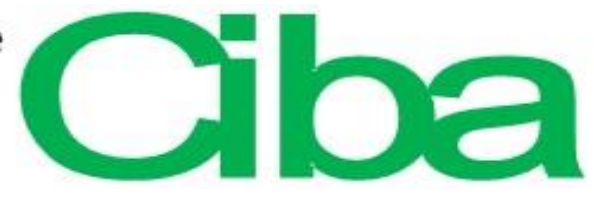

ISSN: 2007 - 9990

\title{
Producción de oxígeno en Pinus teocote Schl. et Cham. y Pinus oocarpa Schiede. En la región Montaña del estado de Guerrero, México
}

Oxygen production of Pinus teocote Schl. et Cham. and Pinus oocarpa Schiede. From the Mountain of Guerrero, Mexico

Produção de oxigênio em Pinus teocote Schl. et Cham. e Pinus oocarpa Schiede. Na região montanhosa do estado de Guerrero, no México

Juan Manuel Ríos Camey*

Universidad Autónoma de Nuevo León, México jmrc_x25@hotmail.com https://orcid.org/0000-0003-4546-0265

*Autor para correspondencia

Oscar Alberto Aguirre Calderón Universidad Autónoma de Nuevo León, México oaguirre16@gmail.com https://orcid.org/0000-0001-5668-8869

Bernardo López López Universidad Intercultural del Estado de Guerrero, México lopez_020986@hotmail.com https://orcid.org/0000-0002-4639-1145

Beatriz Calleja Peláez Universidad Autónoma de Nuevo León, México beatriz.calleja07@gmail.com https://orcid.org/0000-0002-7944-2834 
Revista Iberoamericana de las Ciencias Biológicas

y Agropecuarias

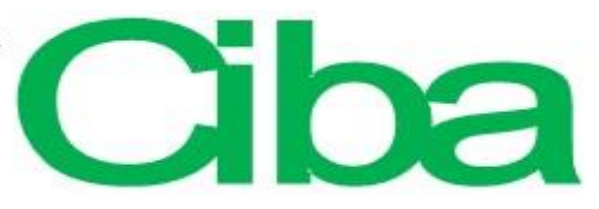

SSN: $2007-9990$

\section{Resumen}

El objetivo de este estudio fue cuantificar la producción de oxígeno de las especies Pinus teocote Schl. et Cham. y Pinus oocarpa Schiede. ubicadas en las áreas forestales de la región de Montaña del estado de Guerrero (México). Para ello, se empleó la ecuación de Schumacher $y i=\exp (b 0+b 1 / x)$, la cual permitió estimar el punto óptimo de producción de oxígeno mediante el cruce del incremento corriente anual (ICA) y el incremento medio anual (IMA). Los resultados muestran que los aumentos en la emisión de oxígeno se pueden predecir adecuadamente a través de la edad, pues se obtuvieron valores de $\mathrm{R}^{2}$ de 0.99 para Pinus teocote Schl. et Cham. y de 0.98 para Pinus oocarpa Schiede. En concreto, se puede indicar que Pinus oocarpa Schiede. produjo $1856 \mathrm{~kg} \mathrm{O}_{2}$ arbol$^{-1}$ a los 71 años, mientras que Pinus teocote Schl. et Cham. solamente emitió $952 \mathrm{~kg} \mathrm{O}_{2}$ arbol $^{-1}$ a los 76 años. Por tal motivo, se puede concluir que la primera especie generó más del doble de oxígeno que la segunda, de ahí que se recomiende su empleo para las plantaciones forestales o reforestaciones de servicios ambientales.

Palabras clave: modelos de crecimiento, oxígeno, Pinus oocarpa Schiede., Pinus teocote Schl. et Cham., servicios ambientales.

\section{Abstract}

The objective of this study was to quantify the oxygen production of the species Pinus teocote Schl. et Cham. and Pinus oocarpa Schiede. located in the forest areas of the Mountain region of the state of Guerrero (Mexico). For this, the Schumacher equationyi $=\exp (b 0+b 1 / x)$ was used, which allowed us to estimate the optimal point of oxygen production by crossing the annual current increase (ICA) and the annual average increase (IMA). The results show that increases in oxygen emission can be predicted adequately through age, since $\mathrm{R}^{2}$ values of 0.99 were obtained for Pinus teocote Schl. et Cham. and from 0.98 for Pinus oocarpa Schiede. In particular, it can be indicated that Pinus oocarpa Schiede. produced $1856 \mathrm{~kg} \mathrm{O}_{2}$ tree $^{-1}$ at age 71, while Pinus teocote Schl. et Cham. only issued $952 \mathrm{~kg} \mathrm{O} 2$ tree $^{-1}$ at age 76 . For this reason, it can be concluded that the first species generated more than twice as much oxygen as the second, which is why its use is recommended for forest plantations or reforestation of environmental services. 
Revista Iberoamericana de las Ciencias Biológicas

y Agropecuarias

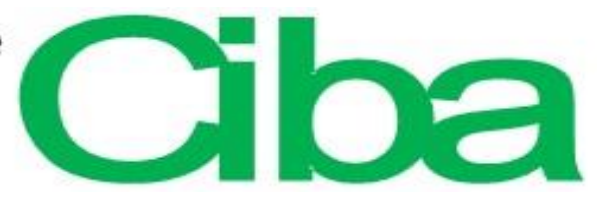

ISSN: 2007 - 9990

Keywords: growth models, oxygen, Pinus oocarpa Schiede., Pinus teocote Schl. et Cham., environmental services.

\section{Resumo}

O objectivo deste estudo foi quantificar a produção de espécies de oxigénio pinus teocote Schl. et Cham. e Pinus oocarpa Schiede. localizado nas áreas de floresta da região montanhosa do estado de Guerrero (México). Por isso, foi usada a equação que permitiu Schumacher estimar a produção óptima de oxigénio através do cruzamento do incremento corrente anual (ICA) e o aumento médio anual (IMA). Os resultados mostram que os aumentos na emissão de oxigénio pode ser adequadamente previsto através dos valores antigos para $\mathrm{R}^{2}$ de 0,99 para pinus teocote $\mathrm{Schl}$ foram obtidos. et Cham. e de 0,98 para Pinus oocarpa Schiede. Em particular, pode ser indicado que Pinus oocarpa Schiede. produziu $1856 \mathrm{~kg} \mathrm{O}_{2}$ arbol $^{-1}$ aos 71 anos, enquanto Pinus teocote Schl. et Cham. emitiu apenas $952 \mathrm{~kg} \mathrm{de}_{2}$ arbol $^{-1}$ aos 76 anos. Portanto, pode-se concluir que a primeira espécie gerado mais de duas vezes o segundo oxigênio, daí o uso de plantações florestais ou reflorestamento de serviços ambientais recomendadas.

Palavras-chave: modelos de crescimento, oxigênio, oocarpa Schiede Pinus, pinus teocote Schl . et Cham., serviços ambientais.

Fecha recepción: Julio 2018

Fecha aceptación: Noviembre 2018 
Revista Iberoamericana de

las Ciencias Biológicas

y Agropecuarias

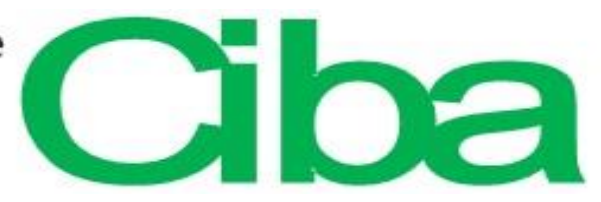

ISSN: 2007 - 9990

\section{Introducción}

Los bosques son ecosistemas constituidos principalmente por árboles, los cuales son determinantes para la conservación de la vida en nuestro planeta, ya que a través del proceso químico de la fotosíntesis generan uno de los ingredientes esenciales para la subsistencia: el oxígeno (Cabudivo, 2017; De Lima, Rojas, Méndez, Salazar y Salmerón, 2017; Pérez-Urria, 2009). Sin embargo, en la etapa final de madurez de esos ecosistemas, la emisión neta de oxígeno disminuye debido a que los árboles solo son capaces de producir la cantidad que requieren para su propia respiración (Azcón, Fleck, Aranda y Gómez, 2008), de ahí que sea determinante conocer cuál es la verdadera capacidad de los bosques para generar dicho elemento durante todas las etapas de su vida, tarea que se puede concretar analizando las concentraciones de carbono en sus diferentes edades (Medina 2010) y mediante el ajuste de modelos de crecimiento en diversas especies forestales (Carillo, 2008).

Por este motivo, el objeto de la presente investigación fue evaluar la capacidad de producción de oxígeno de dos de las especies más abundantes en la región Montaña del estado de Guerrero (México): Pinus teocote Schl. et Cham. y Pinus oocarpa Schiede. (Álvarez, Balboa, Merino y Rodríguez, 2005; Gernandt y Pérez de la Rosa, 2014). La hipótesis nula (Ho) planteada fue la siguiente: existen diferencias entre estas dos especies en cuanto a la generación de oxígeno, lo cual se podría usar como argumento para planificar programas de reforestación que contribuyan a la producción de este elemento y a la valoración de servicios ambientales.

\section{Materiales y métodos}

El área de estudio de la presente investigación se encuentra ubicada entre las siguientes coordenadas geográficas: $98^{\circ} 39^{\prime} 24^{\prime \prime}$ LW y 17¹9' 30" LN (figura 1) a una altitud promedio de 2100 m s. n. m. (Instituto Nacional de Estadística Geográfica e Informática [Inegi, 2016]), y está constituida por los municipios Iliatenco y Malinaltepec, donde crecen de manera natural las especies de pinos mencionadas. Además, tiene un clima templado semicalido, con lluvias intensas en los meses de julio y agosto. La temperatura oscila entre $\operatorname{los}-3^{\circ} \mathrm{C}$ y los $26^{\circ} \mathrm{C}$, mientras que el tipo de suelo en su mayoría es regosol, abundante en materia orgánica (Inegi, 2008; Inegi, 2014). 


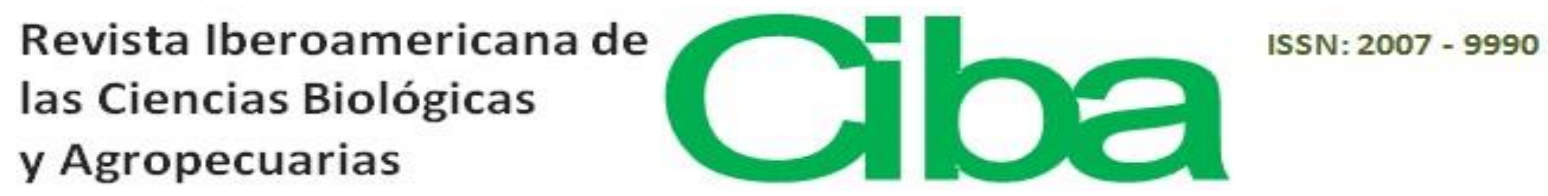

Figura 1. Localización geográfica del área de estudio
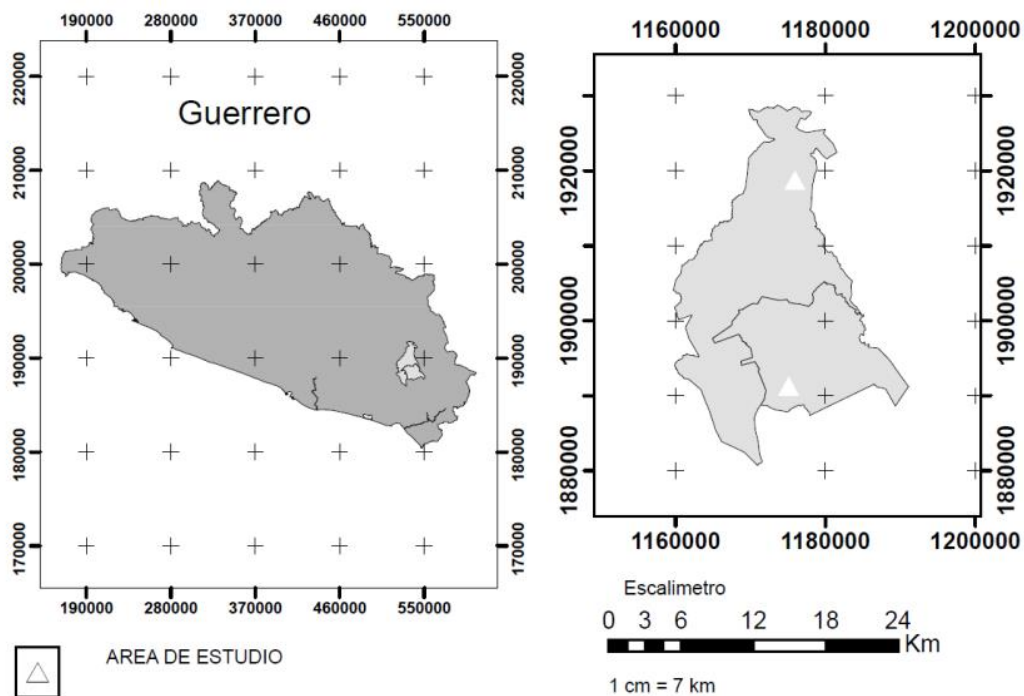

Fuente: Inegi (2016)

\section{Delimitación del área de estudio}

Previo al muestreo, se hicieron recorridos de campo para ubicar las áreas de bosque donde crecen de forma natural las especies Pinus teocote Schl. et Cham. y Pinus oocarpa Schiede. En total, se seleccionaron áreas estratificadas y representativas con presencia de bosques maduros de las mencionadas especies, las cuales abarcan un total de 105 hectáreas. El criterio para la elección de estas fue su amplia distribución en la región Montaña del estado de Guerrero, México.

\section{Selección y derribo de árboles}

Para estimar la producción de oxígeno se escogieron árboles muestra, para lo cual se consideró lo propuesto por Méndez, Luckie, Capó y Nájera (2011), quienes describen los criterios, el número y los árboles a extraer. Posteriormente, se seleccionaron 40 árboles (libres de plagas y enfermedades) de Pinus teocote Schl. et Cham. y Pinus oocarpa Schiede., los cuales tenían todas las categorías de diámetro y altura del área de estudio. La muestra de árboles fue derribada durante dos sesiones en un periodo no mayor a tres meses para evitar variaciones en el follaje y contenido de humedad.

Cada árbol se ubicó y se marcó para su fácil identificación. Luego, antes de ser derribado, se midió en pie para determinar el diámetro normal (DN) a 1.30 m con una cinta diamétrica, la 
Revista Iberoamericana de las Ciencias Biológicas

y Agropecuarias

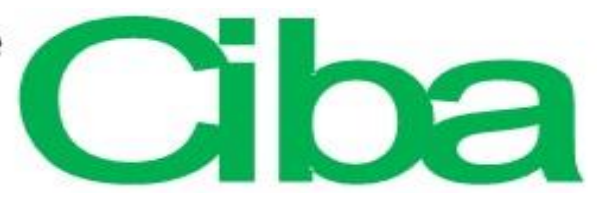

ISSN: 2007 - 9990

altura total en metros (m) y la altura de fuste limpio (AFL) con un clinómetro electrónico Haglof. Esta información se registró en los formatos de campo previamente elaborados (Gayoso, Guerra y Alarcón, 2002; Soriano, Ángeles, Martínez, Plascencia y Razo, 2015). Para evitar daños al arbolado adyacente, se empleó el método de derribo direccional, para lo cual se usó una motosierra Stihl MS 382 de 24". Asimismo, y para evitar la pérdida de componentes vegetales, se colocó una lona sobre el suelo donde cayó el árbol para su posterior seccionado.

\section{Obtención de peso verde}

Derribado el árbol, inmediatamente se separó en dos componentes: hojas-ramas y fuste. El primero de ellos se colocó en costales y se pesó con una báscula digital colgante TecNova-300 kilogramos, la cual tiene una precisión de hasta 100 gramos. Al mismo tiempo, se pesó una muestra representativa de $5 \%$ de hojas-ramas y se empaquetó en bolsas de papel para su traslado al laboratorio. El componente fuste se dividió en trozas: las dos primeras a longitud comercial (2.5 m), y las posteriores a cada metro hasta alcanzar la sección de puntas del árbol, lo cual facilitó el pesado en campo.

De cada troza se obtuvieron rodajas a diferentes alturas de corte, las cuales se pesaron en verde y se obtuvo el diámetro promedio de cada lado de las trozas con una cinta diamétrica Forestry Suppliers FOI0-0106. Las rodajas totales de cada árbol fueron etiquetadas y empaquetadas en bolsas para su traslado al laboratorio. Para obtener el peso verde aéreo de cada árbol se aplicó la siguiente ecuación:

$$
P V A=P C H R+P F+P R
$$

Donde

$P V A=$ peso verde aéreo árbol $(\mathrm{kg})$

$P C H R=$ peso verde de componente hojas-ramas $(\mathrm{kg})$

$P F=$ peso verde fuste $(\mathrm{kg})$

$P R=$ peso verde rodajas $(\mathrm{kg})$. 
Revista Iberoamericana de las Ciencias Biológicas

y Agropecuarias

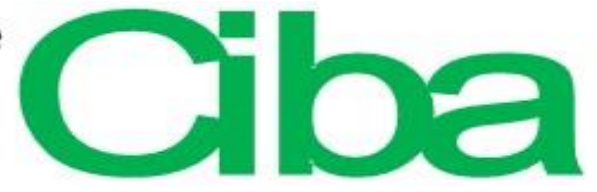

ISSN: 2007 - 9990

\section{Secado y análisis de muestras}

En el laboratorio, las muestras hojas-ramas y rodajas se introdujeron en una estufa de secado (marca Felisa FE-293 de tipo convencional) a una temperatura promedio de $100{ }^{\circ} \mathrm{C} \pm 5{ }^{\circ} \mathrm{C}$ hasta alcanzar peso anhidro, para lo cual se monitoreó el peso de cada muestra cada tercer día con una báscula de alta precisión. Secadas las muestras, se lijaron y se contabilizaron anillos de crecimiento a lo largo de todo el fuste mediante el procedimiento de análisis troncales. Con base en lo anterior, se obtuvieron diferentes edades, diámetros y alturas durante la vida del árbol. Posteriormente se capturó en hoja de cálculo Excel 97-2003.

\section{Cuantificación de biomasa aérea}

La biomasa área de cada componente se obtuvo extrapolando el peso verde aéreo total de campo con el peso verde de muestras y peso seco de muestras de cada componente. Para su obtención se aplicaron las ecuaciones propuestas por Schelegel, Gayoso y Guerra (2000) modificadas:

$$
B A C=\frac{(P V m) *(P V A)}{P V C}
$$

Donde

$B A C=$ biomasa área total árbol ${ }^{-1}(\mathrm{~kg})$

$P V m=$ peso verde muestra de componente $(\mathrm{kg})$

$P V A=$ peso verde aéreo árbol $(\mathrm{kg})$

$P V c=$ peso seco muestra de componente $(\mathrm{kg})$.

\section{Obtención del carbono almacenado}

El carbono (C) almacenado $(\mathrm{kg})$ de cada especie se obtuvo a través del producto de la biomasa aérea por la fracción de carbono estandarizado como $50 \%$ de la biomasa del árbol ( Gayoso et al., 2002; Intergovernmental Panel on Climate Change [IPCC], 2010; Schelegel et al., 2000). 
Revista lberoamericana de las Ciencias Biológicas

y Agropecuarias

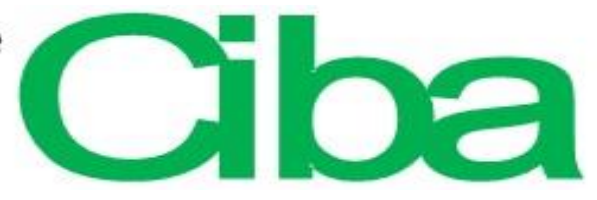

ISSN: $2007-9990$

\section{Estimación de la producción de oxígeno}

La producción de oxígeno está directamente ligada a la acumulación de carbono (C), por lo que Nowak et al. (2007) indican que el oxígeno $\left(\mathrm{O}_{2}\right)$ producido durante la fotosíntesis y consumido durante la respiración se puede estimar con la proporción de pesos atómicos de la molécula de $\mathrm{CO}_{2}$ con el peso del átomo de carbono $(\mathrm{C})$ y oxígeno $\left(\mathrm{O}_{2}\right)$ que lo componen, de este modo:

$O=C * F$

Donde:

$\mathrm{O}=$ producción neta de $\mathrm{O}_{2}(\mathrm{~kg})$

$\mathrm{C}=$ carbono almacenado en la biomasa $(\mathrm{kg})$

$\mathrm{F}=$ factor de conversión a oxígeno tomando como base los pesos atómicos de las moléculas de carbono y oxígeno (32/12).

En tal sentido, se ajustó el modelo ampliamente usado en estudios de crecimiento forestal de Schumacher (López et al., 2017; Murillo et al., 2017; Pacheco, Juárez, Martínez y Ortiz, 2016), el cual se expresa de la siguiente forma:

$$
y=\exp (b 0+b 1 / x)
$$

Donde

$y=$ oxígeno producido en $\mathrm{kg}$

$x=$ edad del árbol en años

$\exp =$ exponencial

bo y $b l=$ parámetros del modelo.

Para estimar el turno absoluto (máxima producción de oxígeno) se obtuvo el incremento corriente anual (ICA) y el incremento medio anual (IMA) en cada especie estudiada, según la siguiente ecuación:

$$
\begin{aligned}
& I C A=\text { diferencia incremento } / \text { diferenciatiempo } \\
& I M A=\text { incremento } / \text { edad }
\end{aligned}
$$


Revista Iberoamericana de las Ciencias Biológicas

y Agropecuarias

Posteriormente, con el modelo ajustado, se derivaron los incrementos en ICA e IMA, $y$ con $x, y$ con $a$, según las fórmulas siguientes:

$$
\begin{aligned}
& I C A=D y / D x=-a / x^{2} \exp (a+b / x) \\
& I M A=D y / D a=-1 / x \quad \exp (a+b / x)
\end{aligned}
$$

Los valores promedios se estimaron mediante la función Proc Means del programa estadístico Statistical Analysis Software System (SAS, 2002), y el ajuste alométrico se realizó con el método de mínimos cuadrados, mediante el procedimiento no lineal Proc Nlin Model del mismo programa.

\section{Resultados y discusión}

Pacheco et al., (2016) ajustaron modelos de regresión para estimar el incremento en una muestra de 20 árboles Pinus montezumae Lamb., dominantes en bosques de Oaxaca. Asimismo, Marroquín, Méndez, Jiménez, Aguirre y Yerena (2018) emplearon una muestra de 50 árboles para la estimación de biomasa y carbono en plantaciones jóvenes de 22 años de edad de Pinus halepensis y Pinus cembroides en Saltillo, Coahuila (México).

En el presente estudio, sin embargo, para estimar la producción de oxígeno se utilizaron 20 árboles de Pinus teocote Schl. et Cham. y Pinus oocarpa Schiede. Este número se consideró adecuado por tratarse de bosques naturales donde se pueden hallar diferentes categorías diamétricas, lo que hubiera implicado, si se hubiese aumentado el tamaño de la muestra, mayores costos en el muestreo, mano de obra y tiempo.

Ahora bien, los valores mínimos para el diámetro normal de los árboles derribados fueron de $5.6 \mathrm{~cm}$ para Pinus teocote Schl. et Cham. y $4.7 \mathrm{~cm}$ para Pinus oocarpa Schiede., con valores máximos de $58.3 \mathrm{~cm}$ y $38.6 \mathrm{~cm}$, respectivamente. En cuanto a la altura total $(\mathrm{m})$, el intervalo fue de entre 6 m y 23 m para Pinus teocote Schl. et Cham., mientras que en Pinus oocarpa Schiede. los valores oscilaron entre $5 \mathrm{~m}$ y $25 \mathrm{~m}$. La edad promedio fue ligeramente superior en Pinus teocote Schl. et Cham., con 9 años respecto a Pinus oocarpa Schiede.; sin embargo, la concentración de oxígeno difirió entre especies, asociado al ritmo de crecimiento de la especie reflejado en diámetro, área basal y volumen (tabla 1). 


\section{Revista Iberoamericana de las Ciencias Biológicas \\ y Agropecuarias}

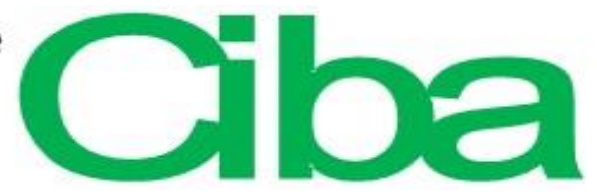

Tabla 1. Parámetros estadísticos descriptivos de Pinus teocote Schl. et Cham. y Pinus oocarpa Schiede. para la región Montaña del estado de Guerrero

\begin{tabular}{lcccc}
\hline \multicolumn{1}{c}{$\begin{array}{c}\text { Estadísticos } \\
\text { (Pinus teocote } \text { Schl. } \text { et } \text { Cham.) }\end{array}$} & DN $(\mathbf{c m})$ & HT (m) & E (años) & O (kg) \\
\hline N. ${ }^{\circ}$ de observaciones & 20 & 20 & 20 & 20 \\
Mínimo & 5.60 & 6.17 & 7 & 5.88 \\
Máximo & 58.3 & 23 & 55 & 610 \\
Media & 28.86 & 17.06 & 30.35 & 248.91 \\
Desviación estándar & 15.34 & 5.59 & 13.39 & 205.68 \\
Coeficiente de variación (\%) & 53.15 & 32.76 & 44.11 & 82.63 \\
Varianza & 235.31 & 31.24 & 179.29 & 42304.26 \\
& & & & \\
Estadísticos & DN $(\mathbf{c m})$ & HT (cm) & E(años) & O(kg) \\
$\quad$ Pinus oocarpa Schiede.) & 20 & 20 & 20 & 20 \\
\hline N. ${ }^{\circ}$ de observaciones & 3.5 & 5 & 4 & 3.49 \\
Mínimo & 38.6 & 25 & 40 & 880.63 \\
Máximo & 20.46 & 15.85 & 21.45 & 288.23 \\
Media & 10.84 & 5.44 & 10.53 & 287.24 \\
Desviación estándar & 52.98 & 34.32 & 49.09 & 99.65 \\
Coeficiente de variación (\%) & 117.50 & 29.5936 & 110.8809 & 82506.81 \\
Varianza & & &
\end{tabular}

Nota: DN = diámetro normal (cm); HT = altura total (m); O = oxígeno producido.

Fuente: Elaboración propia

\section{Estimación de producción de oxígeno}

El modelo de crecimiento forestal de Schumacher en su forma $y=\exp (a+b / x)$ presentó muy buenos ajustes para la predicción de la producción de oxígeno. Se obtuvo un coeficiente de determinación $\left(\mathrm{R}^{2}\right)$ de 0.99 para Pinus teocote Schl. et Cham. y 0.98 para Pinus oocarpa Schiede., con valores de error estándar (Syx) de 0.80 y 1.76, respectivamente; asimismo, ambos modelos alométricos son altamente significativos ( $\mathrm{p}$ < 0.0001), por lo que se puede decir que el modelo predice adecuadamente la producción de oxígeno en cualquier edad del arbolado, es decir, etapas juveniles o maduras del bosque (tabla 2). 
Revista lberoamericana de las Ciencias Biológicas

y Agropecuarias

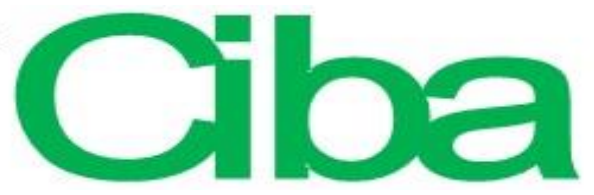

Tabla 2. Estadísticos obtenidos para la predicción de la producción de oxígeno (kg) para Pinus teocote Schl. et Cham. y Pinus oocarpa Schiede. en la región Montaña del estado de Guerrero

\begin{tabular}{cccccccc}
\hline Especie & & Coeficiente & E. E. & Valor de $\boldsymbol{p}$ & $\mathbf{R}^{\mathbf{2}}$ & Sxy & C. V. \\
\hline \multirow{2}{*}{ P. teocote } & $b 0$ & 7.8370 & 0.14 & $<0.0001$ & \multirow{2}{*}{0.99} & 0.80 & 11.46 \\
& $b 1$ & -74.3134 & 2.45 & $<0.0001$ & & & \\
P. oocarpa & $b 0$ & 8.5001 & 0.10 & $<0.0001$ & & & \\
& $b 1$ & -68.1942 & 1.80 & $<0.0001$ & & &
\end{tabular}

Nota: E. E. = error estándar del coeficiente de regresión; $\mathrm{R}^{2}=$ coeficiente de determinación; Sxy = error estándar del modelo de regresión; C. V. = coeficiente de variación; b0, b1 = parámetros de la regresión.

Fuente: Elaboración propia

El modelo exponencial obtenido para la producción de oxígeno en Pinus teocote Schl. et Cham. fue el siguiente:

$$
O=\exp (7.837+(74.3134 / E)
$$

Donde

$\mathrm{O}=$ producción de oxígeno $(\mathrm{kg})$

$\mathrm{E}=$ edad (años).

Asimismo, para Pinus oocarpa Schiede. el modelo alométrico fue el siguiente: $O=$ $\exp (8.5001+(-68.19 / E)$

Donde

$\mathrm{O}=$ producción de oxígeno $(\mathrm{kg})$

$\mathrm{E}=\operatorname{edad}($ años$)$.

En la figura 2 se pueden apreciar los valores observados y predichos del modelo para estimar la producción de oxígeno en Pinus teocote Schl. et Cham. y Pinus oocarpa Schiede. 
Revista Iberoamericana de las Ciencias Biológicas

y Agropecuarias

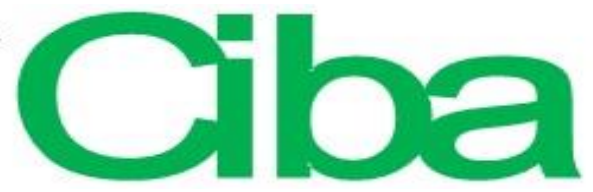

ISSN: 2007 - 9990

Figura 2. Comportamiento de observados y predichos del modelo para predicción de oxígeno: a) Pinus teocote Schl. et Cham., b) Pinus oocarpa Schiede. en la región Montaña del estado de Guerrero (México)
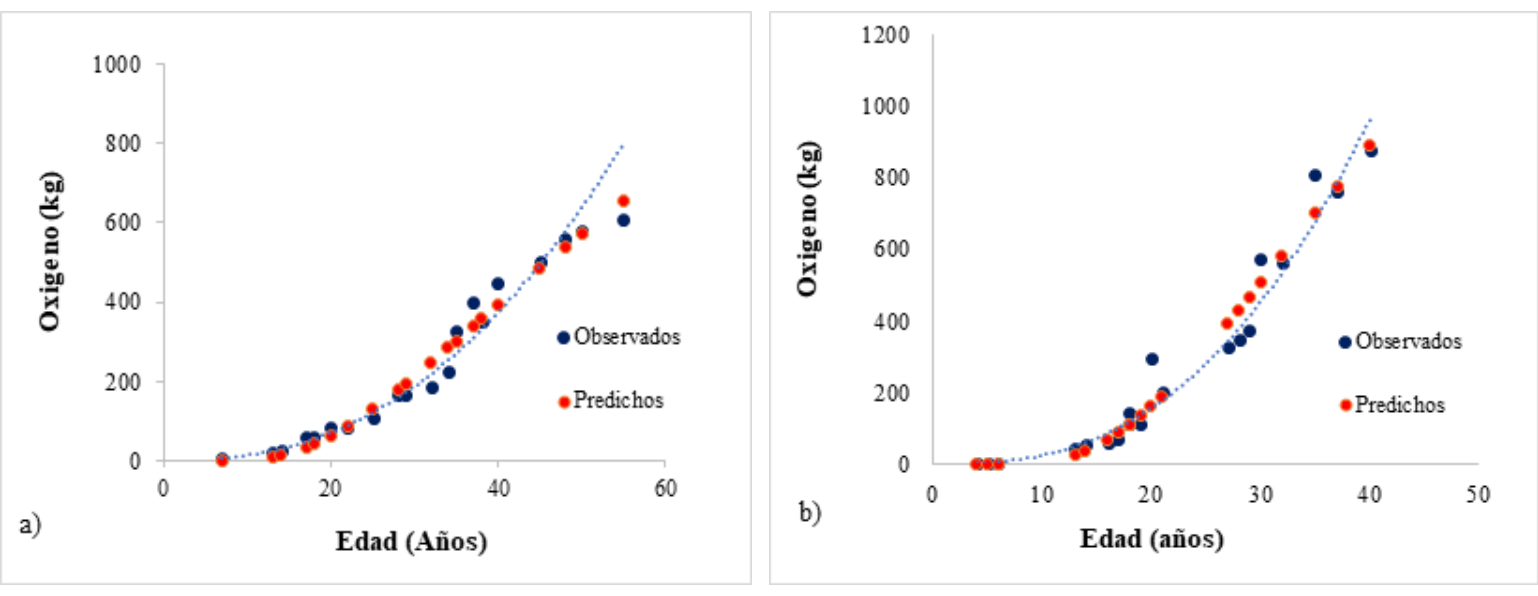

Fuente: Elaboración propia

En este sentido, Guerrero (2013) ajustó el modelo de Schumacher en su forma exponencial para estimar la producción de oxígeno de Pinus cembroides en plantaciones forestales del sureste de Coahuila (México), lo que le permitió conseguir valores de $\mathrm{R}^{2}$ de 0.82 y Syx de 0.96. Resultados similares se encontraron en la presente investigación, donde la edad fue buen predictor de oxígeno para Pinus teocote Schl. et Cham. y Pinus oocarpa Schiede. en la región Montaña del estado de Guerrero. De forma similar, Hernández et al. (2015) ajustaron modelos de crecimiento de alturaedad para Pinus teocote Schl. et Cham. en el estado de Hidalgo, donde el modelo de Schumacher presentó los mejores ajustes con valores de $\mathrm{R}^{2}$ de 0.97 y Syx de 3.34.

Por otra parte, se debe indicar que los parámetros estadísticos del modelo difieren con lo reportado por Pacheco et al. (2016), quienes registraron incrementos en altura para índices de sitio de Pinus montezumae Lamb., con base en la edad del arbolado, ajustando el mejor modelo el de Champan-Richards un $\mathrm{R}^{2}$ adj de 0.94, Sxy de 1517.5 y CME de 12.33; sin embargo, para estudios de biomasa, carbono y, en este caso, producción de oxígeno, se ha corroborado que el modelo exponencial de Schumacher ha conseguido mejores ajustes (López, Méndez, Zermeño, Cerano y García, 2017; Murillo, Domínguez, Martínez, Lagunes y Aldrete, 2017; Pacheco et al 2016). 
Revista Iberoamericana de las Ciencias Biológicas

y Agropecuarias

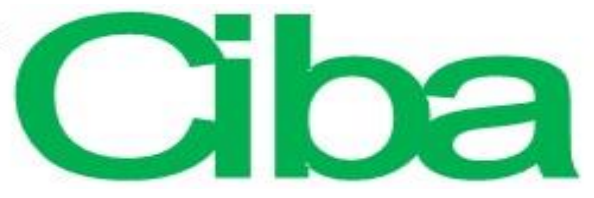

ISSN: 2007 - 9990

\section{Producción de oxígeno e incrementos}

El cruce de ICA e IMA (turno absoluto de máxima producción de oxígeno) ocurrió a los 76 años para Pinus teocote Schl. et Cham. con una máxima generación de oxígeno de $11 \mathrm{~kg} \mathrm{árbol}^{-1}$; mientras que para Pinus oocarpa Schiede. la máxima producción se dio a los 70 años con $26 \mathrm{~kg}$ por árbol ${ }^{-1}$ (figura 3 ).

Figura 3. Producción de oxígeno para a) Pinus oocarpa Schiede. y b) Pinus teocote Schl. et Cham. en la región Montaña del estado de Guerrero
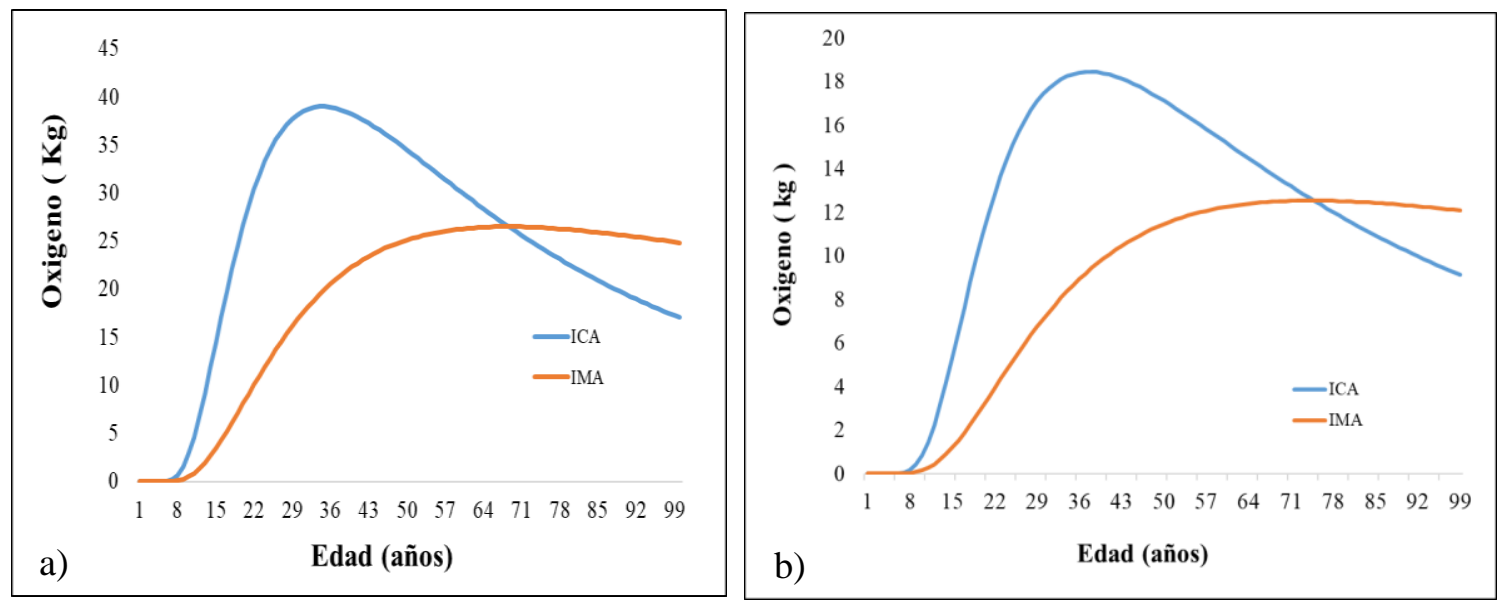

Fuente: Elaboración propia

El máximo ICA obtenido para Pinus teocote Schl. et Cham. fue de $18.4 \mathrm{~kg} \mathrm{de}_{2} \mathrm{año}^{-1}$ a la edad de 38 años, mientras que Pinus oocarpa Schiede. produjo $39{\mathrm{~kg} \mathrm{de} \mathrm{O}_{2} \text { año }}^{-1}$ a la edad de 35 años. Igualmente, es preciso notar que Pinus teocote Schl. et Cham. presentó los mismos aumentos en oxígeno hasta los siete años de edad, mientras que posteriormente el ritmo de crecimiento fue mayor en Pinus oocarpa Schiede. con notables diferencias marcadas en la producción de oxígeno respecto a Pinus teocote Schl. et Cham. (figura 4). 


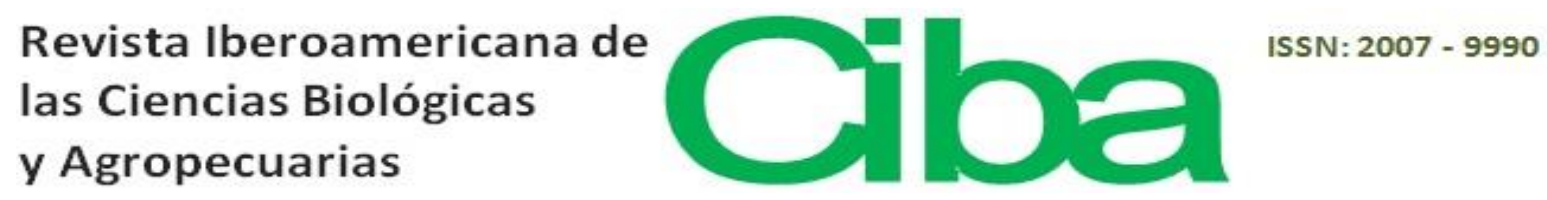

Figura 4. Incremento corriente anual (ICA) de la producción de oxígeno para Pinus teocote Schl. et Cham. y Pinus oocarpa Schiede. en la región Montaña del estado de Guerrero

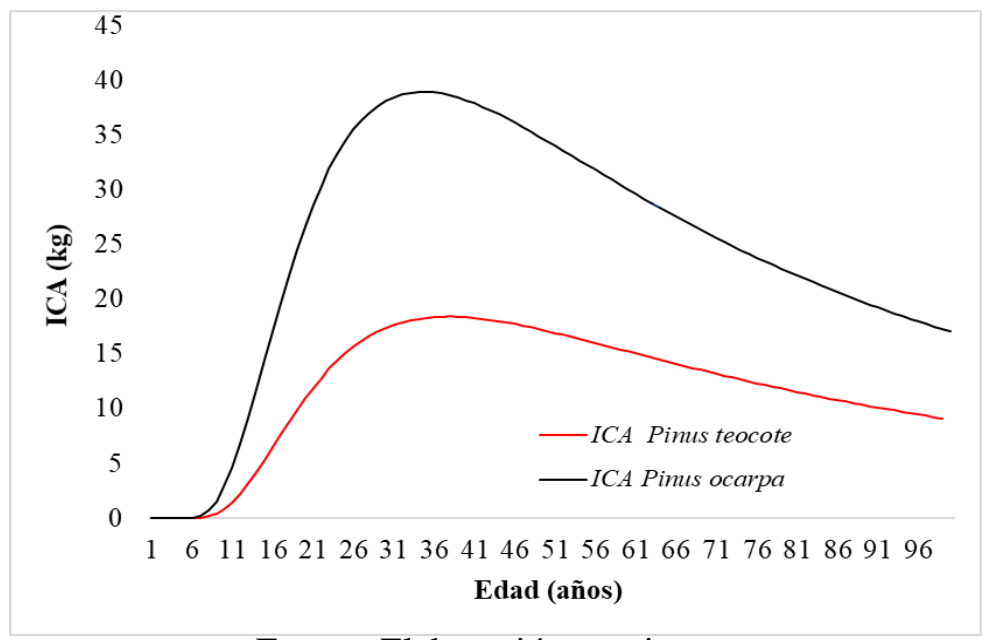

Fuente: Elaboración propia

\section{Producción acumulada de oxígeno}

Considerando el turno absoluto, máxima producción de oxígeno acumulado durante toda la vida del árbol, Pinus oocarpa Schiede. produjo $1856 \mathrm{~kg} \mathrm{O}_{2} \operatorname{arbol}^{-1}$ a los 71 años, mientras que Pinus teocote Schl. et Cham. solamente emitió $952 \mathrm{~kg} \mathrm{O}_{2} \operatorname{arbol}^{-1}$ a los 76 años (figura 5). 


\section{Revista Iberoamericana de las Ciencias Biológicas \\ y Agropecuarias}

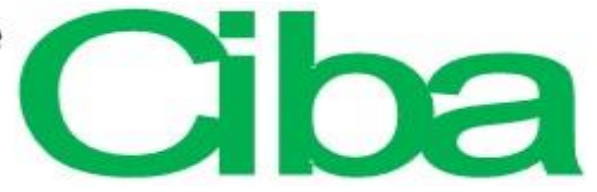

Figura 5. Producción acumulada de oxígeno en Pinus oocarpa Schiede. y Pinus teocote Schl. et Cham. en la región Montaña, Guerrero (México)

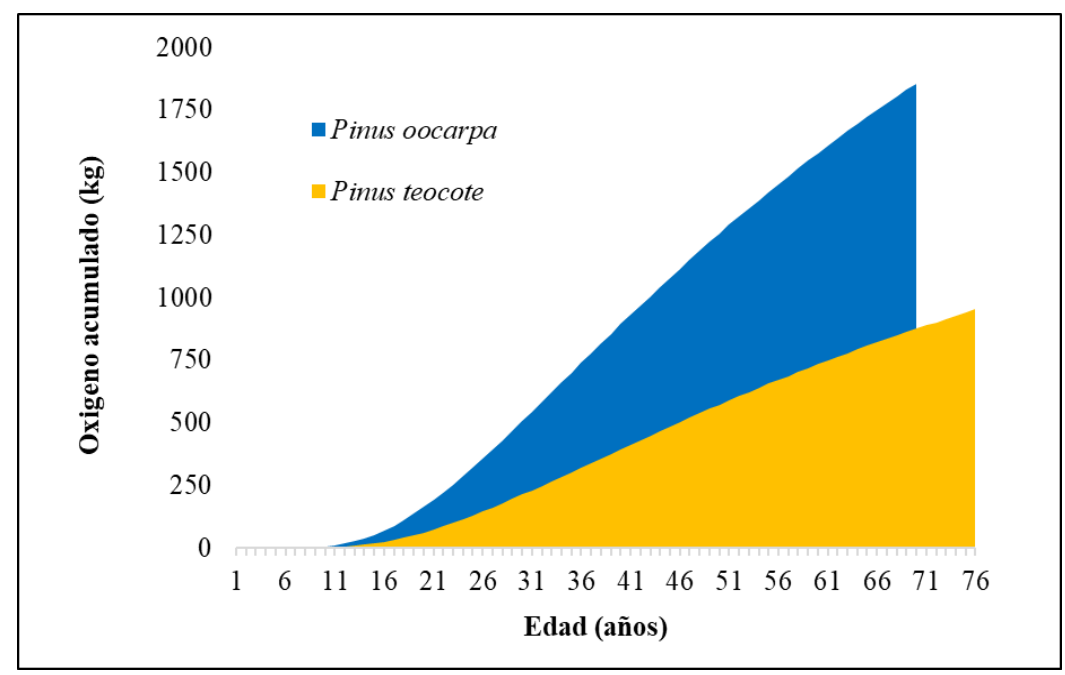

Fuente: Elaboración propia

De acuerdo con Perry y LeVan (2003), una persona consume en promedio $0.84 \mathrm{~kg}$ de oxígeno por día, lo que significa que a partir de los resultados conseguidos se requieren 12 árboles de 70 años de edad de Pinus oocarpa Schiede. y 25 árboles de Pinus teocote Schl. et Cham. para producir el oxígeno diario que requiere una persona (tabla 3).

Tabla 3. Aportación diaria y por persona de oxígeno en Pinus teocote Schl. et Cham. y Pinus oocarpa Schiede. en la región Montaña del estado de Guerrero

\begin{tabular}{cccccc}
\hline Especie & $\begin{array}{c}\text { Turno } \\
\text { absoluto }\end{array}$ & $\begin{array}{c}\text { Oxígeno } \\
\text { acumulado } \\
\text { (kg) }\end{array}$ & $\begin{array}{c}\text { Oxígeno } \\
\text { anual (kg) }\end{array}$ & $\begin{array}{c}\text { Oxígeno } \\
\text { diario (kg) }\end{array}$ & $\begin{array}{c}\text { Arboles } \\
\text { necesarios } \\
\text { /persona/día }\end{array}$ \\
\hline $\begin{array}{c}\text { Pinus oocarpa } \\
\text { Schiede. }\end{array}$ & 70 & $1,855.58$ & 26.50 & 0.072 & 11.57 \\
$\begin{array}{c}\text { Pinus teocote } \\
\text { Schl. et } \text { Cham. }\end{array}$ & 75 & 940.25 & 12.53 & 0.034 & 24.46 \\
\hline
\end{tabular}

Fuente: Elaboración propia

Finalmente, Canfield (2014) indica que la cantidad de oxígeno necesaria por persona es superior cuando la altitud es mayor respecto al nivel del mar ( $40 \%$ más en altitudes mayores a los 3000 m s. n. m.), lo que se debe a la diferencia en la presión atmosférica. Esto significa que se 
Revista Iberoamericana de

las Ciencias Biológicas

y Agropecuarias

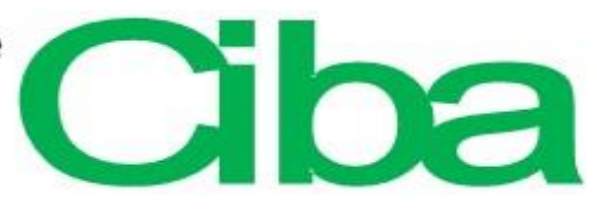

ISSN: 2007 - 9990

requerirían más concentraciones de oxígeno en la atmósfera para abastecer las demandas diarias por persona.

\section{Conclusiones}

Según los datos reportados, se puede concluir que el modelo de crecimiento ajustado predice adecuadamente el oxígeno $(\mathrm{kg})$ con base en la edad del arbolado de las especies estudiadas, pues se hallaron valores de $\mathrm{R}^{2}$ de 0.99 para Pinus teocote Schl. et Cham. y de 0.98 para Pinus oocarpa Schiede.

Asimismo, se comprobó que Pinus oocarpa Schiede. produce más del doble de oxígeno que Pinus teocote Schl. et Cham., por lo que se puede recomendar para proyectos de pagos por servicios ambientales en la región Montaña del estado de Guerrero o en hábitats similares de crecimiento de esta especie.

Por último, se debe promover el cuidado de los recursos forestales, ya que - como se ha constatado - se necesita un número considerable de las especies estudiadas para aportar los requerimientos diarios de oxígeno de una persona.

\section{Agradecimientos}

A la Universidad Autónoma de Nuevo León por el apoyo monetario otorgado para la realización del estudio mediante la beca Conacyt, así como a la Universidad Intercultural del Estado de Guerrero por las facilidades brindadas para el trabajo de campo y laboratorio. 
Revista Iberoamericana de las Ciencias Biológicas

y Agropecuarias

\section{Referencias}

Álvarez, J., Balboa, M., Merino, A. y Rodríguez, R. (2005). Estimación de la biomasa arbórea de Eucalyptus globulus y Pinus pinaster en Galicia. Recursos Rurais, (1), 21-30.

Azcón, J., Fleck, I., Aranda, X. y Gómez, N. (2008). Fotosíntesis, factores ambientales y cambio climático. En Azcón, J., Talón, M. (coord.), Fundamentos de fisiología vegetal (pp. 247263). España: McGraw-Hill.

Canfield, D. E (2014). Oxígeno: una historia de cuatro mil millones de años. Barcelona (España): Editorial Planeta.

Carrillo, E. G. (2008). Casos prácticos para muestreo e inventarios forestales. México: Universidad Autónoma Chapingo. División de Ciencias Forestales.

Cabudivo, C. (2017). Servicio ambiental de secuestro de $\mathrm{CO}_{2}$ y emisión de $\mathrm{O}_{2}$ del bosque natural arboretum el Huayo por niveles de dureza de la madera en Puerto Almendra, Loreto-Perú2015 (tesis de maestría). Universidad Nacional de la Amazonia Peruana. Facultad de Agronomía. Recuperado de http://repositorio.unapiquitos.edu.pe/handle/UNAP/5260.

De Lima, S., Rojas, M., Méndez, J., Salazar, K. y Salmerón, A. (2017). Servicios ecosistémicos de regulación que benefician a la sociedad y su relación con la restauración ecológica. Biocenosis, 31(1-2).

Gayoso, J., Guerra, D. y Alarcón, D. (2002). Contenido de carbono y funciones de biomasa en especies nativas y exóticas: medición de la capacidad de captura de carbono en bosques de Chile y promoción en el mercado mundial. Valdivia: Universidad Austral de Chile.

Gernandt, D. S. y Pérez de la Rosa, J. A. (2014). Biodiversidad de Pinophyta (coníferas) en México. Revista Mexicana de Biodiversidad, 85, 126-133.

Guerrero, L. (2013). Cuantificación de biomasa, carbono y producción de oxígeno en Pinus cembroides Zucc. en Mazapil, Zacatecas, México (tesis profesional). México: Universidad Autónoma Agraria Antonio Narro. Recuperado de http://repositorio.uaaan.mx:8080/xmlui/handle/123456789/1065.

Hernández R., J., García M.., García C. X., Hernández R. A., Muñoz F. H.., y Samperio J. M. (2015). Índice de sitio para bosques naturales de Pinus teocote Schlecht. \& Cham.. en el oriente del estado de Hidalgo. Revista Mexicana de Ciencias Forestales, 6(27), 24-37. 


\section{Revista Iberoamericana de las Ciencias Biológicas y Agropecuarias}

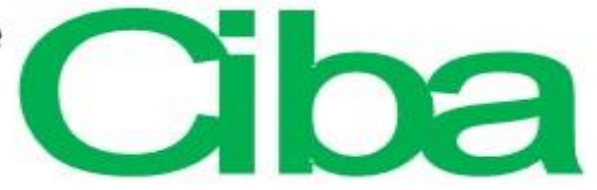

Instituto Nacional de Estadística y Geografía (Inegi) (2008). Conjunto de datos vectoriales. Unidades climáticas. Escala 1:1000 000.n/p.

Instituto Nacional de Estadística y Geografía (Inegi) (2014). Conjunto de datos vectoriales. Perfiles de suelos. Escala 1:1 000 000. n/p.

Instituto Nacional de Estadística y Geografía (Inegi) (2016). Conjunto de datos vectoriales de uso del suelo y vegetación. Serie VI. (Capa Unión). Escala 1:250 000. n/p.

Intergovernmental Panel on Climate Change (IPCC) (2010). Aspectos del cambio climático incluidos su potencial ambiental y sus consecuencias socioeconómicas.

López, J., Méndez, J., Zermeño, A., Cerano, J. y García, M. (2017). Impacto de descortezadores en el incremento radial de Pinus teocote Schiede. ex Schltdl. \& Cham. y Pseudotsuga menziesii (Mirb.) Franco. Revista Mexicana de Ciencias Forestales, 8(41), 82-108.

Marroquín, P., Méndez, J., Jiménez, J., Aguirre, O. y Yerena, J. (2018). Estimación de biomasa aérea en Pinus cembroides Zucc. y Pinus halepensis Mill. en Saltillo, Coahuila. Revista Mexicana de Ciencias Forestales, 9(47), 94-110.

Medina V. J. (2010). La dieta del dióxido de carbono $\left(\mathrm{CO}_{2}\right)$. Conciencia Tecnológica, (39), 50-53.

Méndez, J., Luckie, S., Capó, M. y Nájera, J. (2011). Ecuaciones alométricas y estimación de incrementos en biomasa aérea y carbono en una plantación mixta de Pinus devoniana Lindl. y P. pseudostrobus Lindl., en Guanajuato, México. Agrociencia, 45(4), 479-491.

Murillo, Y., Domínguez, M., Martínez, P., Lagunes, L. y Aldrete, A. (2017). Índice de sitio en plantaciones de Cedrela odorata en el trópico húmedo de México. Revista de la Facultad de Ciencias Agrarias, 49(1), 15-31.

Pacheco, G., Juárez, W. S., Martínez, D. y Ortiz, R. (2016). Análisis del crecimiento e incremento y estimación de índice de sitio para Pinus montezumae Lamb. en Santiago Textitlán, Sola de Vega, Oaxaca. Foresta Veracruzana, 18(2), 21-28.

Pérez-Urria, E. (2009). Fotosíntesis: aspectos básicos. Reduca (Biología), 2(3).

Perry, J. and Le Van, M. D. (2003). Air purification in closed environments: overview of spacecraft systems. Army Natrick Soldier Center. Retrieved from http://nsc.natick.army.mil/jocotas/ColProPapers/Perry-LeVan. 


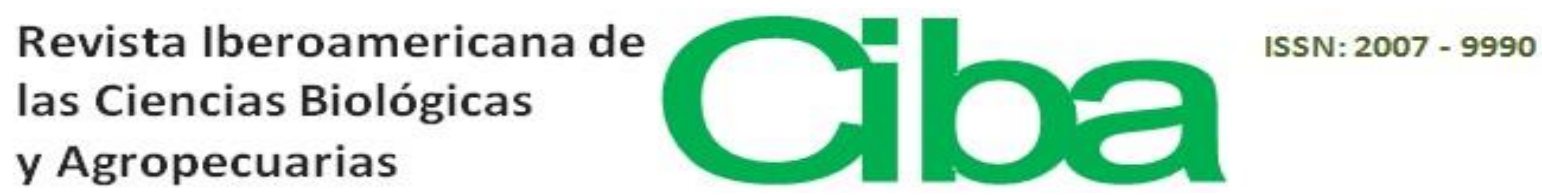

Schelegel, B., Gayoso, J. y Guerra, J. (2000). Manual de procedimientos. Muestreos de biomasa forestal. Medición de la capacidad de captura de carbono en bosques de Chile y promoción en el mercado mundial. Proyecto FONDEF. Universidad Austral de Chile.

Soriano, M., Ángeles, G., Martínez, T., Plascencia, F. y Razo, R. (2015). Estimación de biomasa aérea por componente estructural en Zacualtipán, Hidalgo, México. Agrociencia, 49(4), 423-438.

Statistical Analysis Software System (SAS) (2002). SAS version 9 for Windows. Copyritht @2002 SAS Institute Inc. Cary, NC 27813, USA All. Rights Peserved. 
Revista Iberoamericana de las Ciencias Biológicas

y Agropecuarias

ISSN: 2007 - 9990

\begin{tabular}{|c|c|}
\hline Rol de Contribución & Autor (es) \\
\hline Conceptualización & Juan Manuel Ríos Camey \\
\hline Metodología & Juan Manuel Ríos Camey (Principal) Oscar Alberto Aguirre Calderón (Apoyo) \\
\hline Software & No Aplica \\
\hline Validación & Oscar Alberto Aguirre Calderón (Principal) Juan Manuel Ríos Camey (Apoyo) \\
\hline Análisis Formal & Bernardo López López \\
\hline Investigación & $\begin{array}{l}\text { Juan Manuel Ríos Camey (Principal) Bernardo López López (Apoyo) Beatriz Calleja } \\
\text { Peláez (Apoyo) }\end{array}$ \\
\hline Recursos & $\begin{array}{l}\text { Universidad Autónoma de Nuevo León (Principal) Universidad Intercultural del } \\
\text { Estado de Guerrero ( Ароуо) }\end{array}$ \\
\hline Curación de datos & Juan Manuel Ríos Camey \\
\hline $\begin{array}{l}\text { Escritura - Preparación del borrador } \\
\text { original }\end{array}$ & Juan Manuel Ríos Camey (Principal) Bernardo López López (Apoyo) \\
\hline Escritura - Revisión y edición & Oscar Alberto Aguirre Calderón (Principal) Beatriz Calleja Peláez (Apoyo) \\
\hline Visualización & Juan Manuel Ríos Camey \\
\hline Supervisión & Oscar Alberto Aguirre Calderón (Principal) Beatriz Calleja Peláez ( Apoyo) \\
\hline Administración de Proyectos & Juan Manuel Ríos Camey \\
\hline Adquisición de fondos & $\begin{array}{l}\text { Juan Manuel Ríos Camey, Oscar Alberto Aguirre Calderón, Bernardo López López y } \\
\text { Beatriz Calleja Peláez }\end{array}$ \\
\hline
\end{tabular}

